\title{
- Can Covid-19 Impact Your Oral Health?
}

\section{Shaliga Bhatnagar}

\section{IJCRR}

Section: Healthcare

ISI Impact Factor

(2019-20): 1.628

IC Value (2019): 90.81

$\operatorname{SJIF}(2020)=7.893$

Dentist and Regional Medical Advisor, Abbott India Limited, New Delhi 110075, India.

E-mail: shaliga.bhatnagar@gmail.com

COVID-19, Coronavirus disease-2019 is a communicable disease which is caused by coronavirus. It was found that this pathogen was of a severe acute respiratory syndrome coronavirus- 2 also called SARS-CoV-2. This is the 7th variety of its kind to be infecting humans.

The virus is transmitted from one host to the other via air droplets/direct proximity with oral, nasal, or eye mucous membranes. Multiple studies suggest that COVID-19 takes about 1-2 weeks to incubate and some studies suggest 5 days on an average. The clinical symptoms can be an acute respiratory infection, fever, cough, (WHO guidelines).

As a preventive measure, social distancing and isolation have helped us to avoid contamination at community level in a certain way. However, detecting these symptoms at the right time is very crucial. It has been seen that coronavirus is actually the second most prevalent cause of upper respiratory tract infection in adults and lower respiratory infections and can be combined with systemic symptoms.

Previous studies have failed to identify the co-relation with virus dependant clinical symptoms as viral infections do not generate symptoms, instead, the host's immune system causes them. It has been seen that there are around 60 possible combinations of symptoms among mild COVID-19 patients. Similar to a recently published cohort study, it was found that cough, weakness, and myalgia were the most common symptoms.

23 significant amalgamations of symptoms when comparing men and women were also seen. These findings are substantially important because men might have fewer symptoms. An increased level of acknowledgment and caution among health personnel when addressing an asymptomatic patient is mandatory. Some of the commonly seen oro-facial expression of this viral infection can significantly render to early diagnosis of COVID-19.

Some latest reports have shown that the only leading symptom can be loss of taste and smell. The most prevalent symptoms among all diagnoses patients were cough, runny nose, and weakness in both men and women. Studies suggest that anosmia was recurrent among women and not men.

Olfactory Dysfunction was more common seen from the third to fifth day of the illness, with no such statistical difference in both the sexes. Studies suggest that majority of the cases complained of change in their taste sensation. There was a gross change in the taste bud perception, especially for salty and sweet taste. It was commonly seen amongst women.

Studies suggest that most of infected patients complained of Xerostomia which was strongly associated with a sensation of burning mouth and loss of taste. Studies have clearly shown that facial pain's chief complaint was commonly seen in women, especially in (facial and masticatory muscles). A significant correlation was established between facial pain and nasal congestion.

We know that nasal congestion occurs during upper respiratory infection due to dilatation of veins in the nasal epithelium, which leads to the accumulation of secretions in the sinuses. It causes change in the pressure, which stimulates the trigeminal nerve's endings, leading to sensation of pain. Studies suggest that masticatory muscle pain during mastication. A significant correlation was seen between oro-facial and masticatory pain.

Studies suggest that few patients also reported of change in their tastebuds, few reported plaque-like patterns in the tongue. Some reported inflammatory changes in the oral cavity, palate, tongue, and gingiva. Hence, a strong correlation was established between shift of taste sensation, inflamed palate and plaque-like changes in the tongue.

Few also reported oral bleeding but no spontaneous bleeding was reported. Studies suggest that viral upper respiratory infection is actually one of the most frequently identified cause of olfactory dysfunction. Such impairments are categorized into conductive losses stemming from obstruction of the nasal passages and sensorineural causes from damage to the olfactory neuroepithelium, which are most often credited to post-viral olfactory loss. 
Well reported, the presence of neurologic manifestations among the infected patients who were admitted in Wuhan, China, in the year 2019. Loss of smell and taste was the most commonly reported peripheral nerve symptoms. These findings were based on the previous known cases of severe acute respiratory syndrome and Middle East respiratory syndrome viruses that can enter the central nervous system through a retrograde neuronal route. Since then, many reports have described the same pattern of infection.

Reports suggest that the very first symptom among $25 \%$ of COVID-19 cases is 'anosmia'. As witnessed in the previous studies and now in the current study, 38.3\% reported olfactory dysfunction as the first symptom and $66 \%$ reported the presence of olfactory dysfunction, eventually during the illness period.

To find the high prevalence of olfactory dysfunction, we found a significant correlation with nasal congestion. This clearly reflects that obstructed nasal passages could have served as a significant component to the olfactory impairment. It is also worthy to mention that we found no correlation between nasal congestion and anosmia.

The damage to olfactory neuroepithelium had a significant contribution to anosmia. A real loss of taste is sporadic, as such and it usually leads to the inability to perceive the odor of food due to olfactory dysfunction. $25.8 \%$ reported lost sense of smell with an impaired sense of taste in the absence of other symptoms.
Results have shown the need to examine at the right point in time and isolate patients with olfactory and taste impairment to turn down the COVID-19 infection rate. The main stimulation for saliva formation is the taste and it was seen that most of the infected cases reported dysgeusia and xerostomia. The results showed no significant correlation seen between dysgeusia and nasal congestion or xerostomia with nasal congestion and rhinorrhea.

This condition could be explained by olfactory dysfunction or may suggest neurological involvement that may lead to dysgeusia and xerostomia. SARS-CoV-2 may co-exist with various combinations of symptoms. General symptoms such as cough and weakness $(37.5 \%)$, cough and myalgia (32\%), and myalgia and weakness $(30.5 \%)$ were the most common combinations of symptoms.

The culmination of several studies may lead us to some new clinical information that could increase early diagnosis of COVID-19 patients. It is worthy to learn about differences between sexes, as cough and runny nose were significantly more prevalent among women than among men. It is important to notice the high proportion of patients presented with an impaired sense of smell and taste. Hence, Right detection at Right time with Right measures can be a game changer for all of us. 